Portland State University

PDXScholar

7-22-1994

\title{
Building of a Thermoacoustic Refrigerator and Measuring the Basic Performance
}

Torsten Blumreiter

Portland State University

Follow this and additional works at: https://pdxscholar.library.pdx.edu/open_access_etds

Part of the Physics Commons

Let us know how access to this document benefits you.

\section{Recommended Citation}

Blumreiter, Torsten, "Building of a Thermoacoustic Refrigerator and Measuring the Basic Performance" (1994). Dissertations and Theses. Paper 4714.

https://doi.org/10.15760/etd.6598

This Thesis is brought to you for free and open access. It has been accepted for inclusion in Dissertations and Theses by an authorized administrator of PDXScholar. Please contact us if we can make this document more accessible: pdxscholar@pdx.edu. 


\section{THESIS APPROVAL}

The abstract and thesis of Torsten Blumreiter for the Master of Science in Physics were presented July 22, 1994, and accepted by the thesis committee and the department.

COMMITTEE APPROVALS:

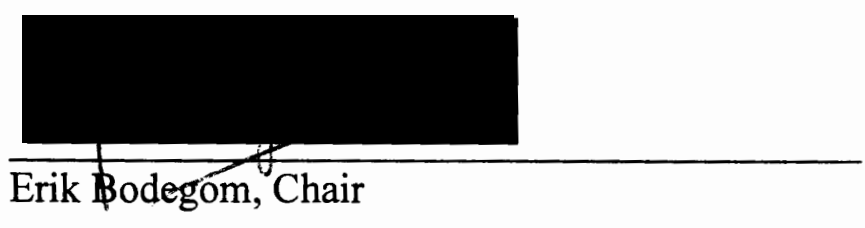

Laird C. Brodie
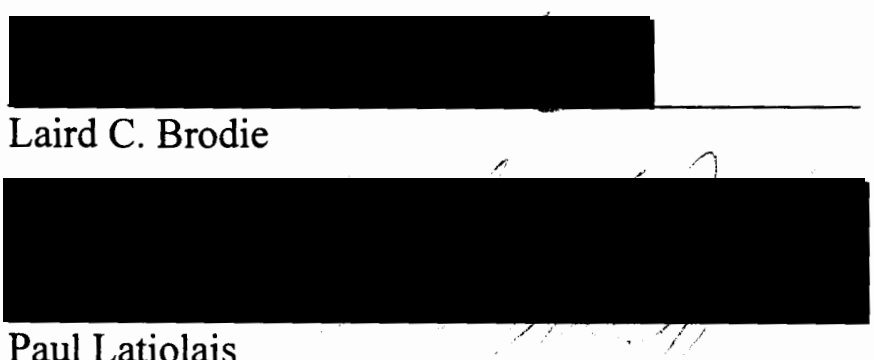

Representative of the Office of Graduate Studies

DEPARTMENT APPROVAL:

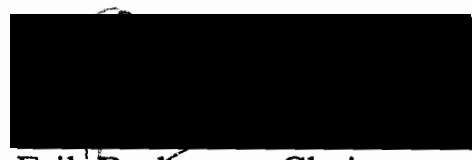

Erik'Bodegom, Chair

Department of Physics 


\section{ABSTRACT}

An abstract of the thesis of Torsten Blumreiter for the Master of Science in Physics presented July 22, 1994

Title: Building of a thermoacoustic refrigerator and measuring the basic performance

The application of thermoacoustic phenomena for cooling purposes has a comparatively short history. However, recent experiments have shown that thermoacoustic refrigeration can achieve practical significance for both every day cooling in households and cryocooling for scientific purposes due to its high reliability, environmental safety and functioning under extreme conditions.

We build a thermoacoustic refrigerator driven by a commercial loudspeaker. It was equipped with a vacuum pump and an entrance port for introducing different gases under different pressures as working fluids. It contained two thermocouples and a pressure transducer for quantitative measurements of the basic performance.

The resonance frequency of the tube for different gases has been determined and compared to the theoretical value. The temperatures of the hot and the cold heat exchanger have been measured.

Also, a simple thermoacoustic oscillator for demonstration purposes was built. After immersing one end in liquid nitrogen or heating up the other end with a bunsen burner it started to oscillate and emit a sound. 
BUILDING OF A THERMOACOUSTIC REFRIGERATOR AND MEASURING

THE BASIC PERFORMANCE

by

TORSTEN BLUMREITER

A thesis submitted in partial fulfillment of the

requirements for the degree of

\section{MASTER OF SCIENCE \\ in \\ PHYSICS}

Portland State University

1994 


\section{Table of Contents}

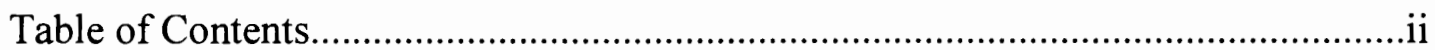

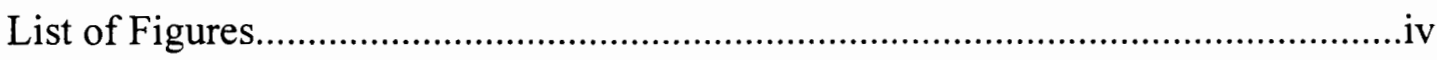

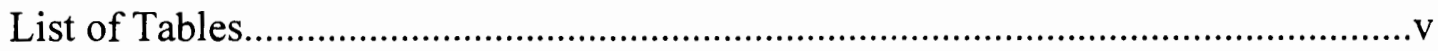

Acknowledgements..............................................................................................vi

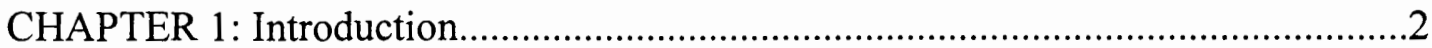

CHAPTER 2: Theory of Thermoacoustic Engines.....................................................4

2.1 Qualitative Description of a Thermoacoustic Refrigerator..........................

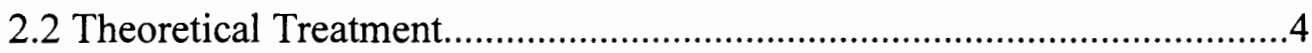

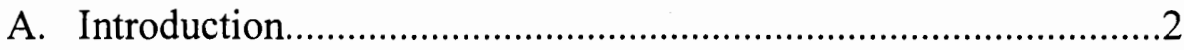

B. Properties of Sound Waves........................................................

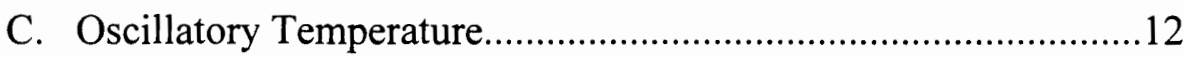

D. Heat Flux, Work Flux and Efficiency........................................16

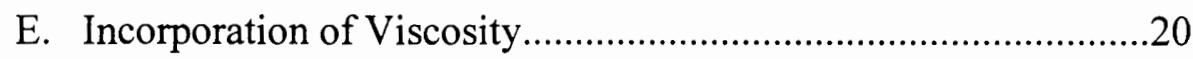

2.3 Summary of Working Conditions and Working Fluids............................22

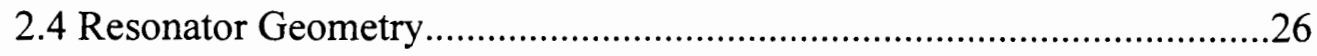

CHAPTER 3: Experimental Setup and Construction................................................28

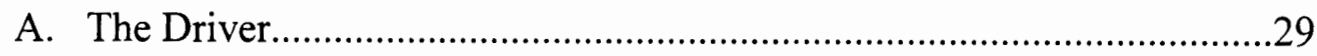

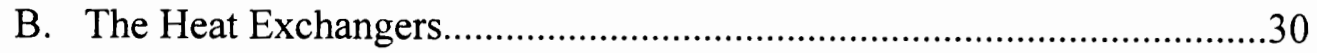

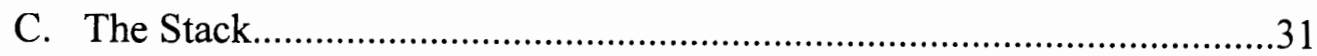

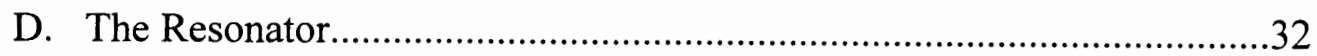

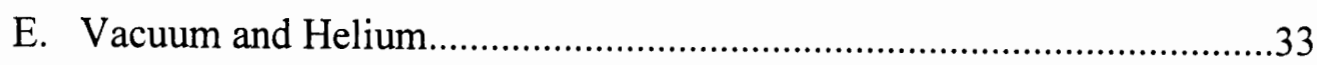

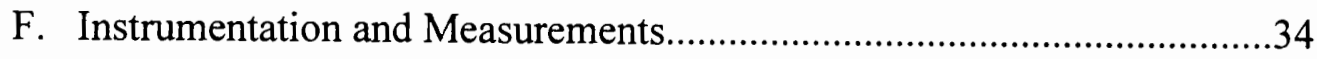

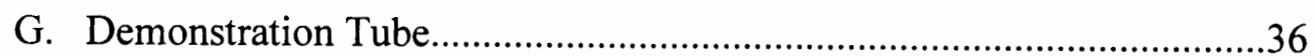


CHAPTER 4: Experimental Results and Conclusions

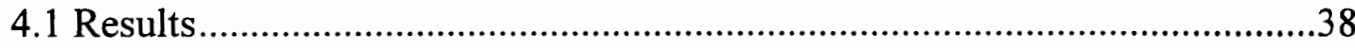

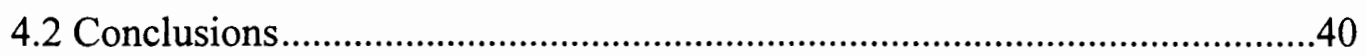




\section{List of Figures}

Figure 1: Sketch of the work principle of a prime mover and a heat pump....................4

Figure 2: Drawing of the pulse tube used in this experiment (units in inch) ...................

Figure 3: Four- step- cycle of a fluid parcel near a plate ................................................

Figure 4: p-V-diagram of a complete cycle .............................................................

Figure 5: The thermoacoustic cycle in a s-T-diagram...................................................

Figure 6: Schematic of four possible resonator geometries ............................................26

Figure 7: Picture of the modified speaker with the aluminum cone replaced the

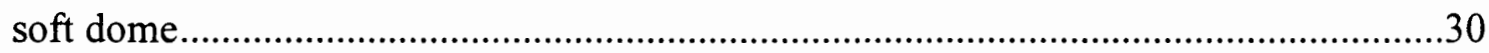

Figure 8: String alignment jig and section of the plastic film..........................................

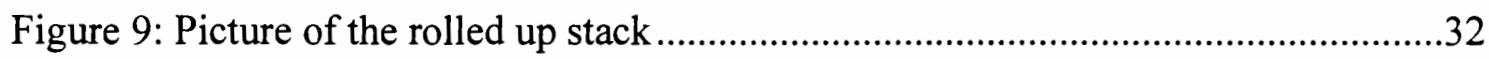

Figure 10: Drawing of the vacuum and helium filling system........................................34

Figure 11: Calibration curve of the thermocouple in conjunction with an amplifier, with one junction in an ice bath and the other junction in water .....................................35

Figure 12: Drawing of a simple thermoacoustic oscillator..............................................36

Figure 13: Voltage signal of the pressure tranducer in arbitrary units as a function

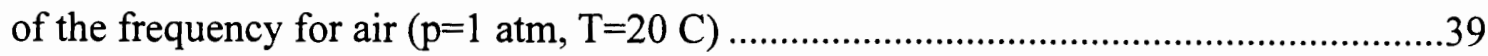

Figure 14: Voltage signal of the pressure transducer in arbitrary units versus the

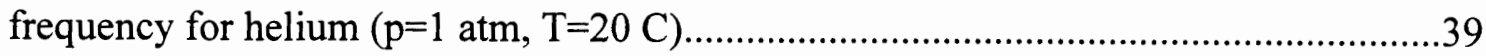




\section{List of Tables}

Table 1: Some thermodynamic properties related to the performance of a thermoacoustic engine of various gases at their resonance frequency in the resonator used in this experiment at the same temperature and pressure $(\mathrm{p}=1 \mathrm{~atm}$,

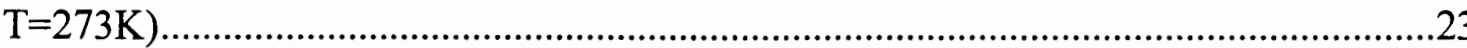

Table 2: Prandtl number and viscous diffusity for various gases for $\mathrm{T}=273 \mathrm{~K} \ldots \ldots \ldots \ldots \ldots . .25$

Table 3: Calculated and experimentally determined resonance frequency for $\mathrm{T}=20 \mathrm{C}$ 


\section{Acknowledgements}

I am very thankful to Mr. Rudolf Zupan from the science support shop for his continuous and fine work.

1 would like to thank Dr. Erik Bodegom, my academic adviser, for his many useful suggestions.

I would like to acknowledge the flexibility of the committee members Laird C. Brodie, Paul Latiolais and Erik Bodegom. 


\section{CHAPTER 1: Introduction}

Thermoacoustics is a branch of physics dealing, as the word already reveals, with thermodynamics and acoustics. Specifically, it relates to the transfer of heat, work, enthalpy, and other thermodynamic quantities and to the conversion of heat energy into other kinds of energy and vice versa by using acoustic phenomena or sound waves'. In this research, thermoacoustics is applied to a refrigerating system in which sound waves are used for transferring heat from a location of lower temperature to one of higher temperature (heat pump). The usage of sound waves has some significant advantages compared to current refrigerating systems. The only moving part is the sound generating part, a voice coil of a regular commercial loudspeaker. This coil can be driven quite inexpensively by a simple amplifier. This amplifier needs only to amplify one sinusoidal frequency, the resonance frequency of the refrigerator. Good acoustic insulation can make the refrigerator fairly quiet. It is also an environmentally significant step to safe technology, because no chlorofluorocarbons (CFCs) are released.

The history of thermoacoustics goes back to the glassblowers in the 18th and 19th century. Sometimes, while blowing their glass tubes, a loud sound occurred. First experiments were probably done by Byron Higgins in 1777, who was able to excite organ pipe oscillations in a large tube, open at both ends, by suitable placement of a hydrogen flame inside. The best known thermoacoustical devices in history are the Sondhauss tube and the Rijke tube, introduced in 1850 and 1859 , respectively. The Soundhauss tube was an open tube of about $15 \mathrm{~cm}$ made of glass with a sphere on top. After heating the closed end or spherical end it started to produce and maintain a vibration.

Probably, the best known example of the excitation of sound waves is known as "Taconis oscillations" that occur when a tube, closed on the top, is inserted in a liquid helium dewar. They were systematically observed by Clement and Gaffney and recently some 
quantitative experiments were done by Yasaki².

The history of serious and systematic research of the reverse process is quite short. The first experiments were done by Gifford and Longsworth ${ }^{2}$. They operated the Sondhauss tube in reverse, i.e. they produced a temperature difference by applying a low- frequency, high- amplitude acoustic wave to the gas in a tube. They called their device a pulse tube. Later experiments were done by a group of researchers in Los Alamos, California in the eighties. Their experiments resulted in an almost practical refrigerator, and theory and experimental results ${ }^{3}$ were compared.

The Russian scientist E.I. Mikulin ${ }^{4}$ used a different technique. He outfitted the original pulse tube with an orifice. This orifice allows the gas to expand through the orifice into a large reservoir. This increased the refrigeration capacity considerably. According to Rott ${ }^{1}$, the creation of the field of theoretical thermoacoustics goes back to Kirchhoff in 1868. In 1896, Lord Rayleigh discussed, although more qualitatively in nature, the phase difference between temperature and motion. The most complete quantitative theory was introduced and developed by Rott and co- workers in the sixties and seventies. 


\section{CHAPTER 2: Theory of Thermoacoustic Engines}

\subsection{Qualitative Description of a Thermoacoustic Refrigerator}

When talking about conversion of heat into work, two concepts always appear: prime mover and heat pump (see figure 1 for a schematic representation of both engines indicating the flow of energy). A prime mover requires a cold and hot heat exchanger and by transferring heat from the hot to the cold exchanger, work can be done. (That was the way the glass blowers generated their sounds and the way the Sondhauss tube worked.) The reverse device called heat pump transfers heat from the cold to the hot heat exchanger by absorbing work. Both machines typically work in cycles and in a steady state which means that the state of the heat exchangers after one cycle has not changed.

Prime mover

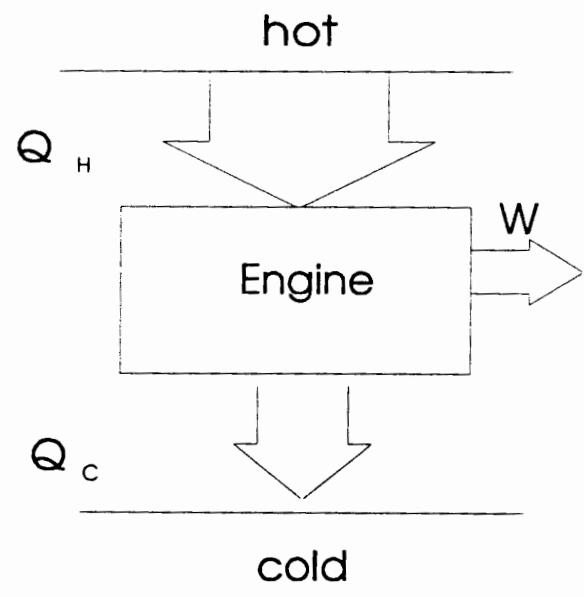

Heat pump

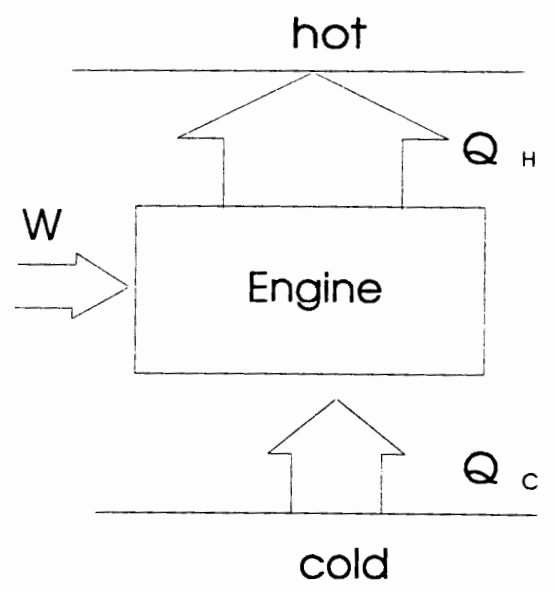

Figure 1: Sketch of the work principle of a prime mover and a heat pump

The acoustic refrigerator (pulse tube) is a heat pump that absorbs acoustic power 
introduced by a loudspeaker or a piston into a resonator and produces and maintains a temperature gradient across the tube. In figure 2 the main parts of the pulse tube that we built can be seen.

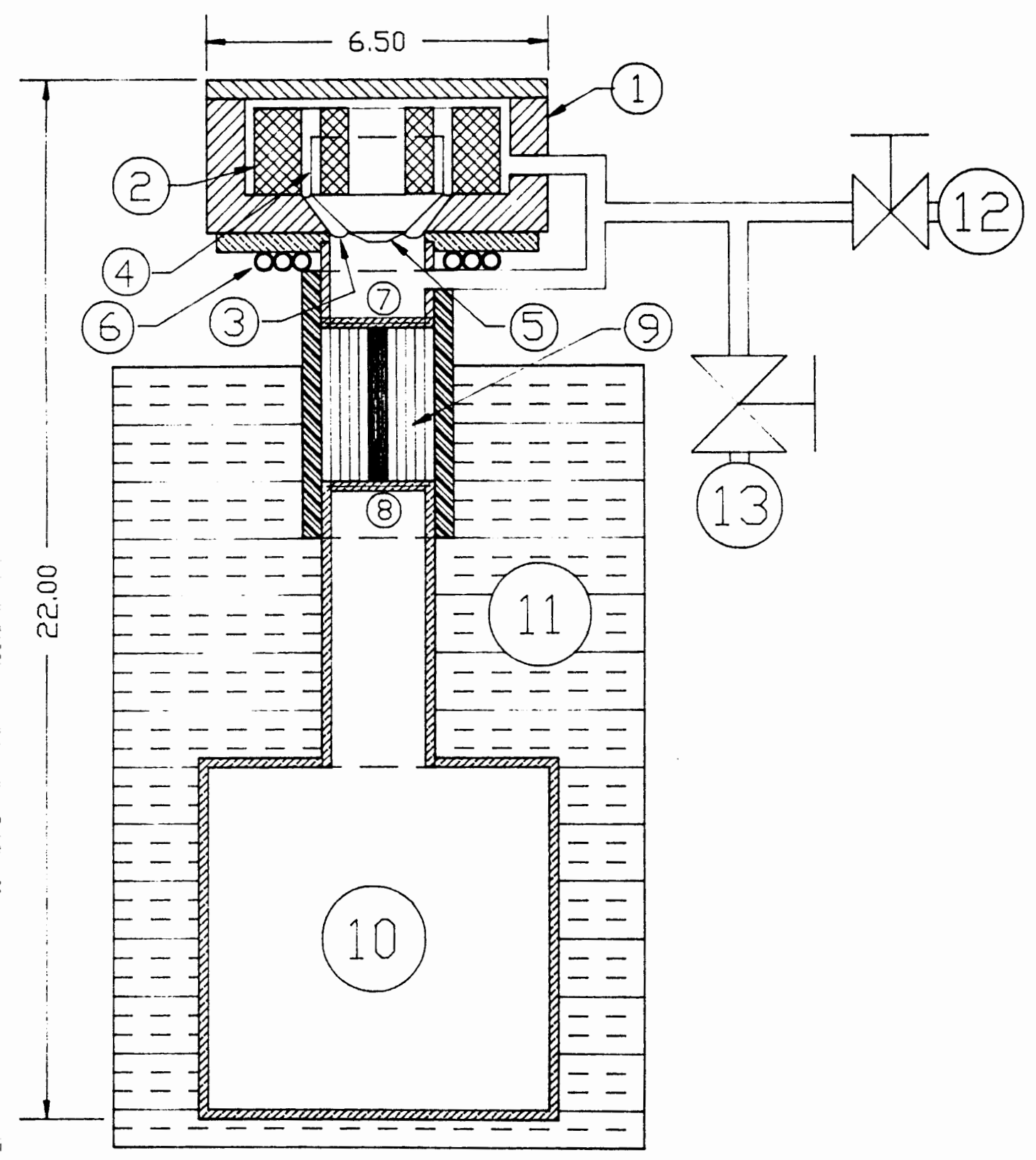

Figure 2: Drawing of the pulse tube used in this experiment (units in inch) 
The labeling numbers mean the following:

1. -speaker housing

2. -magnet

3. - extra surround

4. -voice coil

5. -aluminum cone

6. -cooling tubes

7. -hot heat exchanger

8. -cold heat exchanger

9. -stack

10. -cold section

11. -insulation

12. -vacuum pump valve

13. -helium regulator valve

The essential parts are the speaker on top ( 2 and 4$)$, the stack of poor heat conducting plates (9) between two heat exchangers ( 7 and 8 ) and the resonator. The speaker maintains a standing acoustic wave in the resonator. The principle of thermoacoustic refrigerators can be understood by considering small fluid parcels moving back and forth between a state of compression and expansion. In an undisturbed standing wave no heat transfer and work absorption would occur, except for dissipative losses. This is changed, however, in the presence of a second medium. To illustrate, a complete cycle that a fluid parcel goes through near a plate, is shown in four steps in figure 3. 


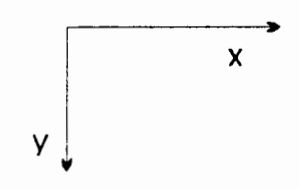

a)
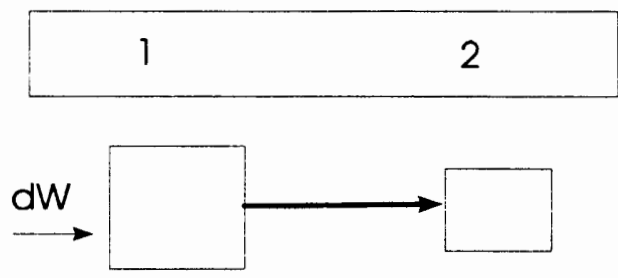

c)
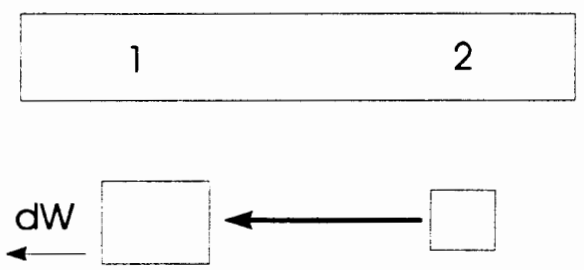

b)

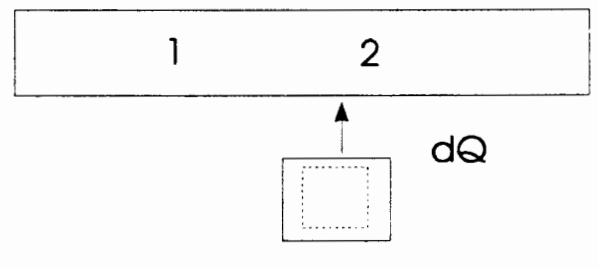

d)

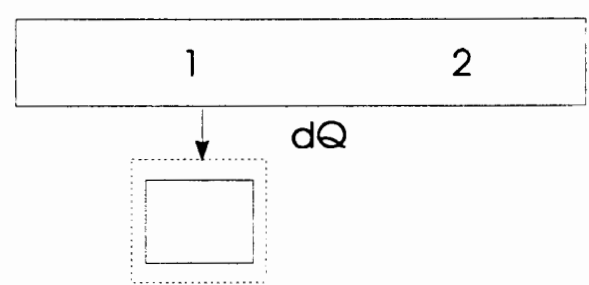

Figure 3: Four- step- cycle of a fluid parcel near a plate

Step a) shows the adiabatic compression of the parcel due to increased pressure resulting in the associated movement to the right, a decrease in volume and increase in temperature. At the farthest point on the right hand side, heat transfer from the heated parcel to the plate takes place. This results in a volume and temperature decrease of the parcel. Step c) describes the adiabatic expansion due to decreased pressure. As the parcel moves to the left it cools down further and expands. Lastly, step d) completes the cycle. Since the temperature has decreased below the plate's temperature, heat is being transferred from the plate to the parcel. It is accompanied by an increase in volume and temperature. Considering a complete cycle, heat is being transferred from the left to the right hand side as well as work being done in the expanded state and absorbed in the 
compressed state. Since the temperature in the compressed state is higher, net work has been absorbed. In figure 4 , the cycle is shown in a p-V-diagram.

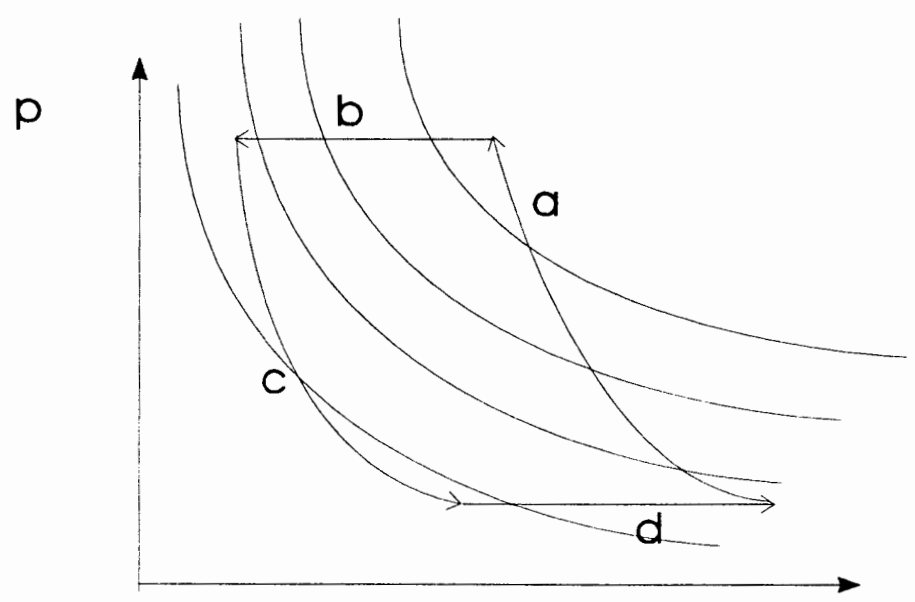

Figure 4: p-V-diagram of a complete cycle

The heat flow is equivalent to an entropy flow. Steps a) and c) are nearly adiabatic, therefore the entropy of the parcel remains constant. In position 1 in figure $3 \mathrm{~d}$, heat is being picked up from the plate which results in an entropy increase of $\Delta s$. This amount is carried from the position 1 to position 2 where it is returned to the plate. Hence, in half a cycle entropy of $\Delta s$ has moved from the left to the right. 


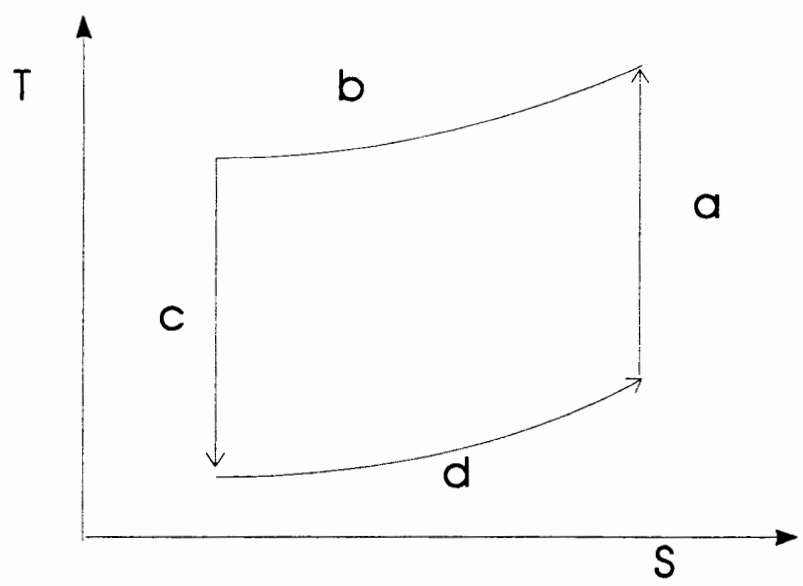

Figure 5: The thermoacoustic cycle in a s-T-diagram

In practice, the displacement of the fluid parcel is small in comparison with the length of the plate. Therefore, the heat is not transferred in one step from the cold to the hot heat exchanger but carried gradually across the plate. The heat deposited by one parcel at a certain location of the plate is picked up by the next one and moved further and so forth until it reaches the hot heat exchanger where it is being removed by cooling water tubes. As already mentioned, the whole process can run in the reverse direction. This occurs if the temperature gradient along the plate is large enough. The engine will work as a prime mover, i.e. the fluid parcels start to oscillate spontaneously. Heat will be transferred from the hot to the cold heat exchanger and work in the form of the generation of sound waves is done. The description of this cycle is the same as the heat pump but the cycle is completed in the opposite direction and energy fluxes have reversed signs.

\subsection{Theoretical Treatment}

\section{A. Introduction}

Since fluids have only a negligible resistance to shear force, sound waves in fluids occur 
only longitudinally, i.e., the direction of propagation is the same as the movement of the molecules carrying the wave. The molecules move irregularly and randomly. In the presence of a sound wave an averaged directed motion in the direction of the wave propagation takes place. This causes a change in pressure and hence a backdirected force towards the original state. The usual treatment of sound waves is macroscopic. This means that individual molecular motions are neglected and only a macroscopic fluid parcel, yet small enough to be treated as an infinitesimal, is considered.

As long as the displacement of the molecules is proportional to the backdirected force, we speak of harmonic waves. This leads to a linear differential equation describing the behavior of the wave. If the areas of equal phase and amplitude are planes, perpendicular to the direction of propagation, the wave is said to be plane.

In acoustics, the pressure and the oscillatory velocity of the fluid parcel are often used to describe the sound wave. We assume that the amplitude of the sound wave is small compared to the average pressure. We will assume that all oscillating quantities (such as temperature, entropy) are small and non- linear terms can be ignored. Or, in mathematical terms, every quantity is expanded in a power series and second and all higher order terms are neglected. These variation terms are designated by a subscript 1 while the equilibrium quantities are designated by subscript 0 .

The complex notation for physical quantities is consequently used for the calculations, although the physics is described just by the real part.

\section{B. Properties of Sound Waves}

The one dimensional wave equation for the pressure $p_{1}$ is:

$$
\frac{\partial^{2} p_{1}}{\partial x^{2}}=\frac{1}{c^{2}} \frac{\partial^{2} p_{1}}{\partial t^{2}}
$$


with $c=\sqrt{\left(\frac{\partial p}{\partial \rho}\right)_{\rho=\rho_{0}}}$ defined as the phase velocity of the sound wave. The most simple solution is described by the trigonometric functions using the customary complex Eulernotation and convenient boundary conditions:

$$
p_{1}=p_{A} e^{\mathrm{i}(k x-\omega t)}
$$

whereas $\omega$ is angular frequency of the wave. From Euler's equation for fluids ${ }^{6}$, describing a relationship between the pressure of the sound wave and the oscillatory velocity $v_{1}$ of a parcel, and the well known relation of the phase velocity

$$
c=\lambda f=\frac{\omega}{k}
$$

the following expression for $v_{1}$ is obtained:

$$
v_{1}=\frac{1}{\rho_{0}} \int \frac{\partial p}{\partial x} \mathrm{~d} t=\frac{p_{A}}{\rho_{0} c} e^{\mathrm{i}(k x-\omega t)}
$$

In our experiments we deal exclusively with standing waves. In a pipe, standing wave patterns are built up due to superposition of the initial and the reflected wave. Thus, the pressure at a certain point can be calculated just by adding up the pressures of the back and forth traveling wave, or:

$$
p_{1}=p_{1}^{+}+p_{1}^{-}=p_{A}\left(e^{\mathrm{i}(k x-\omega t)}+e^{\mathrm{i}(-k x-\omega t)}\right)
$$

This can be simplified to:

$$
p_{1}=2 p_{A} \cos k x e^{-i \omega t}
$$

The oscillatory velocity in the standing wave is obtained by substituting (5) in (3) as follows: 


$$
v_{1}=\mathrm{i} \frac{2 p_{A}}{\rho_{0} c} \sin k x e^{-\mathrm{i} \omega t}
$$

Considering only the real part of either formula, it can be seen that the spatial and the temporal part of a standing wave are not coupled anymore. Also, unlike the traveling wave, there is a constant phase shift of $\pi / 2$ between the pressure and velocity for both the temporary and the spatial part as indicated by the imaginary unit and the different trigonometric functions. It follows that the pressure antinode is at a location where the velocity node is and vice versa.

\section{Oscillatory Temperature}

The next step is to derive a relationship between the pressure and the temperature distribution in the bulk gas for a standing wave. The temperature is considered to consist of a mean temperature $T_{0}$ and a small variation in the $\mathrm{x}$-direction $T_{1}$ (the linear term of the power series expansion) and to be constant in $y$ - direction. With the assumption of small pressure and temperature variations, assuming an adiabatic process, $T_{1}$ can be written as

$$
T_{1}=\left(\frac{\partial T}{\partial p}\right)_{\mathrm{s}} p_{1}
$$

By using the well-known Maxwell relation? we obtain:

$$
T_{1}=-\frac{1}{\rho_{0}^{2}}\left(\frac{\partial \rho}{\partial s}\right)_{s} p_{1}
$$

which can be reworked to:

$$
T_{1}=-\frac{1}{\rho_{0}^{2}}\left(\frac{\partial \rho}{\partial T}\right)_{p}\left(\frac{\partial T}{\partial s}\right)_{p} p_{1}=\frac{T_{0} \beta}{\rho_{0} c_{p}} p_{1}
$$


with $c_{p}=\frac{1}{m}\left(\frac{\partial H}{\partial T}\right)_{p}=\frac{T_{0}}{m}\left(\frac{\partial S}{\partial T}\right)_{p}$ the isobaric heat capacity ${ }^{8}$

and $\beta=\frac{1}{V}\left(\frac{\partial V}{\partial T}\right)_{p}=-\frac{1}{\rho_{0}}\left(\frac{\partial \rho}{\partial T}\right)_{p}$

the thermal expansion coefficient at constant pressure9.

All those relationships are derived by expanding the partial derivatives and by applying the general chain rule and using the Maxwell relations.

For an ideal gas, we obtain:

$$
\left(\frac{\partial T}{\partial p}\right)_{s}=\frac{T_{0} \beta}{\rho_{0} c_{p}}=\frac{\gamma-1}{\gamma} \frac{T_{0}}{p_{0}}
$$

by using the adiabatic equation for an ideal gas

$$
T p^{\frac{1-\gamma}{\gamma}}=\text { constant. }
$$

So far, we only considered the waves in the bulk phase of the gas.

By introducing a plate as a second medium, those simple relations change considerably. So far, we have no $y$ - dependence of the temperature, no heat transfer takes place and pressure and temperature are in phase. The second medium causes a thermal lag between temperature and motion in the fluid. To calculate the temperature distribution in the presence of a plate, simplifications must be made.

1) Viscosity is neglected. This assumption is not realistic but simplifies the calculation considerably. For that reason it is going to be added to the final result by means of an estimate.

2) The amplitudes of pressure and velocity are assumed to be constant all over the plate since the plate is short enough with respect to the wavelength.

3) Heat transfer due to thermal conduction occurs only in y-direction and is neglected 
in the $\mathrm{x}$ direction (see the definitions of the directions in figure 3 ) both through the plate and the fluid. This approximation can be made in the fluid since the heat flux due to convective heat flow is much larger and faster, and in the plate since the material of the plate is chosen to be poor with respect to thermal conduction.

4) All mean physical quantities, e.g. $T_{0}, \mathrm{p}_{0}, \mathrm{~s}_{0} \ldots$ are constant in space and time.

5) Beside the mean physical quantities and the first order terms, all higher order terms are neglected.

6) From now on, the exponential time factor $e^{i \omega t}$ that all oscillating quantities have in common will be suppressed. i $v_{1}$ will be replaced by $v_{1}$ for convenience.

One starts with the general equation of heat transfer ${ }^{10}$ :

$$
T \rho\left(\frac{\partial s}{\partial t}+v \bullet \nabla s\right)=\nabla \bullet(K \nabla T)
$$

where $\mathrm{s}$ is the entropy per unit mass and $\mathrm{K}$ the thermal conductivity.

This equation describes the change of entropy at a given point due to convective flow of entropy and thermal conduction of heat. Quadratic terms of the velocity describing entropy changes due to viscosity are neglected according to the assumptions mentioned before. The entropy is considered to consist of a constant part $\mathrm{s}_{0}$ plus a small variation $\mathrm{s}_{1}$ according to the introduced notation. This assumption as well as the existence of only an $\mathrm{x}$-component of the velocity and the neglect of thermal conduction in $\mathrm{x}$ - direction simplifies equation (13) as follows:

$$
\rho_{0} T_{0}\left(\frac{\partial s}{\partial t}+v_{1} \frac{\partial s}{\partial x}\right)=K \frac{\partial^{2} T}{\partial y^{2}}
$$

To eliminate the derivatives of $\mathrm{s}$, the generalized chain rule for the two- dimensional case is used: 


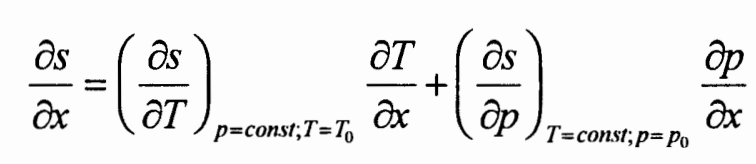

and a similar expression for the time derivative. With equation (10a) and (10b) and one of the Maxwell equations ${ }^{11}$ we obtain:

$$
\begin{aligned}
& \left(\frac{\partial s}{\partial T}\right)_{p=c o n s s ; T=T_{0}}=\frac{c_{p}}{T_{0}} \text { and } \\
& \left(\frac{\partial s}{\partial p}\right)_{T=c o n s s ; p=p_{0}}=\frac{1}{\rho_{0}^{2}}\left(\frac{\partial \rho}{\partial T}\right)_{p=c \text { cons }: ;=\rho_{0}}=-\frac{\beta}{\rho_{0}}
\end{aligned}
$$

Substituting (15), (16), (17) into (14), a differential equation for the temperature $T_{1}$ is obtained.

$$
\mathrm{i} \omega \rho_{0} c_{p} T_{1}-K \frac{\partial^{2} T_{1}}{\partial y^{2}}=\mathrm{i} \omega T_{0} \beta p_{1}-\rho_{0} c_{p} \frac{\partial T_{0}}{\partial x} v_{1}
$$

The solution for this equation with boundary conditions that $T_{1}$ near the plate assumes the temperature of the plate and far away from the plate is still finite, is ${ }^{12}$ :

$$
T_{1}=\left(\frac{T_{0} \beta}{\rho_{0} c_{p}} p_{1}-\frac{1}{\omega} \frac{\partial T}{\partial x} v_{1}\right)\left(1-e^{-\frac{(1+\mathrm{i}) y}{\delta_{k}}}\right)
$$

with $\delta_{k}=\sqrt{\frac{2 K}{\omega \rho_{0} c_{p}}}$ the thermal penetration depth, the characteristic length for thermal diffusion between a gas and a solid boundary. Equation (19) describes the temperature behavior of the fluid at a certain location $\mathrm{x}$ with respect to the plate's temperature. If the fluid is far enough away from the plate, $y>>\delta_{k}$, the exponential term can be neglected and equation (19) becomes 


$$
T_{1, y \propto \delta_{k}}=\left(\frac{T_{0} \beta}{\rho_{0} c_{p}} p_{1}-\frac{1}{\omega} \frac{\partial T}{\partial x} v_{1}\right) .
$$

The first term is identical with equation (10) and the second term is due to the temperature gradient in $\mathrm{x}$ - direction throughout the fluid. If the temperature gradient approaches a critical value, the second term will completely compensate the first term, i.e. fluid and plate have the same temperature at every location. This turns out to be the boundary between working as a refrigerator and a prime mover.

$$
\left(\frac{\partial T}{\partial x}\right)_{\text {crit }}=\frac{T_{0} \beta \omega p_{1}}{\rho_{0} c_{p} v_{1}}=\nabla T_{c r i t}
$$

As well as for the other quantities, the actual temperature distribution is described by the real part of formula (19). The imaginary part, however, has significance for the work and heat flux and is therefore shown explicitly in the next equation:

$$
\operatorname{Im}\left(T_{1}\right)=\left(\frac{T_{0} \beta}{\rho_{0} c_{p}} p_{1}-\frac{1}{\omega} \frac{\partial T}{\partial x} v_{1}\right) e^{-\frac{y}{\delta_{k}}} \sin \frac{y}{\delta_{k}}
$$

\section{Heat Flux, Work Flux and Efficiency}

As is customary in hydrodynamics, the convective flow of a physical quantity can be found out by considering a large volume of fluid and a small cylinder of the fluid carrying a certain amount of that quantity and entering the large volume through a surface element $\mathrm{dA}$ with a velocity $\mathrm{v}_{1}$. Then, in a time $\Delta t$, the length $\mathrm{v} \Delta \mathrm{t}$ of the cylinder will have crossed the surface. Hence, the total heat load entering the volume is

$$
\Delta Q=\int \Delta t \rho_{0} T_{0} v_{1} s_{1} d A
$$

by using the relation ${ }^{13}$

$$
\mathrm{d} q=T \rho \mathrm{d} s=T_{\mathrm{o}} \rho_{\mathrm{o}} s_{\mathrm{l}}
$$


with $\mathrm{s}$ the entropy per unit mass and $\mathrm{q}$ the heat per unit volume. The heat flux in steady state is obtained by dividing equation (23) by $\Delta t$ and time averaging. The surface element in this particular case is the width of the plate $\Pi$ times $d y$.

$$
\dot{Q}=\Pi \int T_{0} \rho_{0}\left\langle s_{1} v_{1}\right\rangle \mathrm{d} y
$$

The integrand in formula (24) is the heat flux density $\dot{q}$.

$$
\dot{q}=T_{0} \rho_{0}\left\langle s_{1} v_{1}\right\rangle
$$

$s_{1}$ can be expressed in terms of $T_{1}$ and $p_{1}$ by using the formula for the total differential and equation (16) and (17). For the time averaged product the well-known relation ${ }^{14}$

$$
\langle A B\rangle=\frac{1}{2} \operatorname{Re}\left(A B^{*}\right)
$$

with the asterix designating the complex conjugate can be used. After performing the previous explained steps and considering that $\left\langle p_{1} v_{1}\right\rangle=0$, for the heat flux density in $\mathrm{x}$ direction is obtained:

$$
\dot{q}=\frac{1}{2} \rho_{0} c_{p} \operatorname{Im}\left(T_{1}\right) v_{1}
$$

After applying equation (24) to carry out the integral, the total heat flux ${ }^{15}$ is obtained to be

$$
\dot{Q}=\frac{1}{4} \Pi T_{0} \delta_{k} \beta p_{1} v_{1}(\Gamma-1)
$$

where $\Gamma$ is the ratio between the temperature gradient and the critical temperature gradient.

This formula reveals the following, partly expected results:

The heat flux is proportional to the area $\Pi \delta_{k}$. It is also proportional to the product of the pressure and the oscillating velocity amplitudes. Since those are $1 / 4 \lambda$ apart the maximum 
heat transfer occurs right in the middle of the pressure and velocity antinodes. Lastly, the heat flux decreases as the temperature gradient approaches the critical temperature gradient. The heat flux density is greatest with respect to the y-direction when the imaginary part of $T_{1}$ is greatest. This occurs about a penetration depth away from the plate (cf. equation (21a)).

To calculate the absorbed net work rate, the work per unit volume done by a differential volume as it expands from $\mathrm{V}$ to $\mathrm{V}+\mathrm{dV}$ is to be considered.

$$
\mathrm{d} w=-p \frac{\mathrm{d} V}{V}=\frac{p}{\rho} \mathrm{d} \rho
$$

Divided by the total time differential it yields:

$$
\frac{d w}{d t}=\frac{p}{\rho} \frac{d \rho}{d t}
$$

For $\mathrm{p}$ in the power series expansion the first approximation $\left(p=p_{0}+p_{1} e^{\mathrm{i} \omega t}\right)$ can be substituted and the total time derivative of $\rho$ in equation (28) can be expanded.

$$
\frac{d \rho}{d t}=\frac{\partial \rho}{\partial t}+(v \bullet \nabla) \rho=i \omega \rho_{1}+v_{1} \frac{\partial \rho_{0}}{\partial x}
$$

After performing the two latter steps, four terms occur in (28). Three of the four terms vanish after time averaging since $\left\langle p_{1}\right\rangle=\left\langle v_{1}\right\rangle=\left\langle p_{1} v_{1}\right\rangle=0$. The following remains

$$
\dot{w}=\frac{\omega}{\rho_{0}}\left\langle i p_{1} \rho_{1}\right\rangle
$$

as the average acoustic power produced per unit volume. $\rho_{1}$ can be expanded in terms of $T_{1}$ and $p_{1}$ by using the formula for the total differential and after computing the various derivatives, the following formula for the acoustic power absorbed per unit volume is obtained: 


$$
\dot{w}=-\omega \beta\left\langle i p_{1} T_{1}\right\rangle=\frac{1}{2} \omega \beta p_{1} \operatorname{Im}\left(T_{1}\right)
$$

This formula shows, similar to the heat flux, that the work done or absorbed is greatest a penetration depth away from the plate (see formula (21a)). It can be integrated over the whole space to obtain the total time averaged acoustic power absorption ${ }^{16}$ :

$$
\begin{aligned}
& \dot{W}=\int_{(V)} \dot{w} d V=-\Pi \Delta x \int_{0}^{\infty} \frac{1}{2} \omega \beta p_{1} \operatorname{Im}\left(T_{1}\right) d y \\
& \dot{W}=\frac{1}{4} \Pi \delta_{k} \Delta x \frac{T_{0} \beta^{2} \omega}{\rho_{0} c_{p}} p_{1}^{2}(\Gamma-1)
\end{aligned}
$$

whereby $\Delta x$ is the length of the plate. As can be seen the total acoustic power absorbed is proportional to the volume generated by the plate surface and the penetration depth, to the square of the pressure amplitude and to the temperature gradient factor $(\Gamma-1)$ that already appeared in the heat flux.

The thermal efficiency of a prime mover is defined as the ratio of the work done and the heat load of the hot heat exchanger.

$$
\eta=\frac{W}{Q_{H}}=\frac{\dot{W}}{\dot{Q}_{H}}
$$

For a heat pump, an equivalent so-called COP (coefficient of performance) is defined just as the reciprocal value.

$$
C O P=\frac{Q_{H}}{W}=\frac{\dot{Q}_{H}}{\dot{W}}
$$

By dividing equation (26) and (33) the COP for this application can be obtained.

$$
C O P=\frac{v_{1} \rho_{0} c_{p}}{\Delta x \beta \omega p_{1}}
$$

With formula (21) and the definition for the $\Gamma$ it can be written 


$$
C O P=\frac{T_{0}}{\Delta x \nabla T_{\text {crit }}}=\frac{\Gamma T_{0}}{\Delta x \nabla T_{0}}=\frac{\Gamma T_{0}}{\Delta T}=\Gamma C O P_{\text {camot }}
$$

with $\mathrm{COP}_{\text {carnot }}$ the $\mathrm{COP}$ of Carnot cycle. It says that the $\mathrm{COP}$ of the pulse tube is simply the COP of the reversed Carnot cycle multiplied by the ratio of the critical and the actual temperature gradient. The Carnot's COP is approached when the critical temperature gradient is approached and the efficiency is less otherwise. That mirrors a common problem in thermodynamics, since the Carnot cycle is a quasistatic cycle and hence takes an infinite time to complete, its efficiency can only be approached when the pumped heat approaches 0 . The faster a cycle is completed and thus the more heat is pumped the less is the efficiency.

\section{E. Incorporation of Viscosity}

A more sophisticated model ${ }^{17}$ that addresses the points which were neglected in previous section uses the most general theory of thermoacoustic engines known so far. In addition to the general equation of heat transfer (13), the equation of motion for fluids ${ }^{18}$ (NavierStokes equation) and the continuity equation must be used. The whole calculation is quite difficult and requires many assumptions, simplifications and approximations to come up with a reasonable interpretable result. Swift ${ }^{19}$ incorporates thermal conductivity in $\mathrm{x}$ - direction in both the plate and the fluid, viscosity and a plurality of plates. From the Navier- Stokes equation, a wave equation for the pressure amplitude and hence for the velocity amplitude in terms of material properties and geometry is obtained. With equation (13), an expression for the temperature distribution is calculated. An expression for the enthalpy flux is obtained by using the law of conservation of energy $y^{20}$ and the work flux in the same way as carried out in this paper. The result, including viscosity but neglecting longitudinal thermal conductivity, making the boundary layer approximation 
$\left(y_{0} \gg \delta_{k}\right)$ and assuming a short plate is shown in the next two formulas ${ }^{21}$ :

$$
\begin{aligned}
& \dot{H}=-\frac{1}{4} \delta_{k} \Pi \frac{T_{0} \beta p_{1}\left\langle v_{1}\right\rangle}{1-\sqrt{\sigma}}(\Gamma-1)-\Pi\left(y_{0} K\right) \frac{d T_{0}}{d x} \\
& \dot{W}=\frac{1}{4} \Pi \delta_{k} \Delta x \frac{(\gamma-1) \omega p_{1}^{2}}{\rho_{0} a^{2}}(\Gamma-1)-\frac{1}{4} \Pi \delta_{v} \Delta x \omega \rho_{0}\left\langle v_{1}\right\rangle^{2}
\end{aligned}
$$

where $\left\langle v_{1}\right\rangle$ is the averaged velocity over the $y$-direction. (In that model, the velocity has gotten an additional $\mathrm{y}$ - component ${ }^{22}$.)

$\dot{H}$ is the enthalpy flux (includes both heat and work flux), $\sigma$ the Prandtl number defined as

$$
\sigma=\frac{c_{k} \mu}{K}=\left(\frac{\delta_{v}}{\delta_{k}}\right)^{2}
$$

with $\mu$ the viscosity, $\mathrm{K}$ the thermal conductivity and $c_{\mathrm{p}}$ the isobaric heat coefficient.

$$
\delta_{v}=\sqrt{\frac{2 \mu}{\omega \rho_{0}}}
$$

is the fluid's viscous penetration depth. It can be interpreted as a characteristic length of interaction between gas and a solid boundary for momentum diffusion. $y_{0}$ is the plate's spacing. The Prandtl number is a dimensionless quantity describing the amount of momentum diffusion relative to thermal diffusion or in other words viscosity to thermal conductivity.

Some additional results for practical usage can be extracted from the two latter equations. The second term in the equation (39) is the power dissipated by viscous shear in the fluid of the boundary layer. Therefore, gases with low viscosity and hence a low Prandtl number work best. Due to dissipative losses, the idea of a critical temperature gradient has to be replaced by a critical temperature range bordered by either one of the conditions 
$\dot{W}=0$ and $\dot{Q}=0$. In that range, only losses occur and heat is transferred from the hot to the cold heat exchanger while the net work is negative.

\subsection{Summary of Working Conditions and Working Fluids}

The theoretical treatment yields some qualitative statements concerning geometry, the choice of the working fluid and thermodynamic conditions for the best possible performance of the refrigerator.

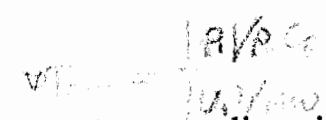
It is desirable to have a large heat transfer because the critical temperature gradient is approached faster and a larger volume can be cooled. The work absorbed should also be large. In equation (26a) and (33), and (38) and (39), respectively, it can be seen how the working conditions and the properties of the working fluid affect the performance of the refrigerator. All these equations contain either the volume spanned by the plate surface and the thermal penetration depth or the area spanned by the width of the plate and the thermal penetration depth. In either case, the plate surface should be made large and the working fluid should have a large thermal penetration depth. One can see from table 1 that helium and hydrogen have the greatest thermal penetration depth. 
Table 1: Some thermodynamic properties ${ }^{23}$ related to the performance of a thermoacoustic engine of various gases at their resonance frequency in the resonator used in this experiment at the same temperature and pressure ( $p=1 \mathrm{~atm}, \mathrm{~T}=273 \mathrm{~K})$

\begin{tabular}{|c|c|c|c|c|c|}
\hline Gas & resonance \\
frequency $\omega$ & $\begin{array}{c}\text { Thermal co- } \\
\text { nductivity } \mathrm{K} \\
\text { in } 10^{-6} \\
\mathrm{~kW} / \mathrm{mK}\end{array}$ & $\begin{array}{c}\text { Density } \rho \text { in } \\
\mathrm{kg} / \mathrm{m}^{3}\end{array}$ & $\begin{array}{c}\text { Specific } \\
\text { heat } \mathrm{c}_{\mathrm{p}} \text { in } \\
\mathrm{kJ} / \mathrm{kgK}\end{array}$ & $\begin{array}{c}\text { Thermal } \\
\text { penetration } \\
\text { depth } \delta_{\mathrm{k}} \text { in } \\
\mathrm{mm}\end{array}$ \\
\hline helium & 4410 & 150.7 & 0.179 & 5.19 & 0.27 \\
\hline oxygen & 1443 & 26.6 & 1.431 & 0.92 & 0.17 \\
\hline nitrogen & 1526 & 26.1 & 1.251 & 1.04 & 0.16 \\
\hline air & 1512 & 26.0 & 1.287 & 1.00 & 0.16 \\
\hline neon & 1987 & 48.1 & 0.894 & 1.03 & 0.23 \\
\hline hydrogen & 5867 & 186.7 & 0.044 & 14.2 & 0.32 \\
\hline argon & 1406 & 17.8 & 5.85 & 0.52 & 0.09 \\
\hline
\end{tabular}


The pressure and velocity amplitudes should be large. This can be achieved by increasing the power generated by the speaker or the by placing the stack right in the middle of the resonator $(1 / 8 \lambda)$ where the product of velocity and pressure amplitudes is greatest. However, according to equation (21), the achievable temperature gradient is maximum at the location of greatest acoustical impedance (pressure antinode). Therefore, the stack in our experiment is moved closer to the driver.

The volume expansivity $\beta$ is generally large for gases. According to equation (38) and (39), working fluids with a low Prandtl number and a small viscous diffusity increase work and heat flux since dissipative losses decrease. Hence, the viscosity and the thermal conductivity (cf. eqn. (40) and (41)) should be small and large, respectively.

If in the equation (38) and (39) the formulas for the pressure and velocity amplitude are plugged in and the definition for the sound velocity is used it can be seen that the sound velocity and the mean pressure should be as large as possible.

There is another practical reason for the sound velocity in the working fluid to be large. The loudspeaker runs much better at higher frequencies and should not run below its resonance frequency $(325 \mathrm{~Hz})$.

Again, helium and hydrogen show good properties with respect to the requirements to thermal conductivity, viscosity, and sound velocity (see tables 1 and 2). 
Table 2: Prandtl number and viscous diffusity for various gases for $T=273 \mathrm{~K}$

\begin{tabular}{|c|c|c|c|}
\hline Gas & $\begin{array}{c}\text { Viscosity } \mu \text { in } 1^{-7} \\
\mathrm{~kg} / \mathrm{ms}^{24}\end{array}$ & $\begin{array}{c}\text { viscous penetration } \\
\text { depth } \delta_{v} \text { in } \mathrm{mm}\end{array}$ & Prandtl number $\sigma$ \\
\hline helium & 194.1 & 0.22 & 0.668 \\
\hline oxygen & 201.8 & 0.14 & 0.697 \\
\hline nitrogen & 175 & 0.135 & 0.697 \\
\hline air & 182.7 & 0.14 & 0.702 \\
\hline neon & 311.1 & 0.19 & 0.665 \\
\hline hydrogen & 87.6 & 0.26 & 0.666 \\
\hline argon & 221 & 0.073 & 0.644 \\
\hline
\end{tabular}




\subsection{Resonator Geometry}

The basic pulse tube is a quarter wavelength tube with one open end and one closed end. The velocity and displacement antinodes are at the open end and the pressure antinode is at the closed end. The system is driven from the open end and the heat flux in the heat pump mode is directed towards the opposite end, i.e. the cold heat exchanger is nearer to the driver (figure 6a). In that assembly, the heat generated by the driver would interfere with the cold produced at the cold heat exchanger.

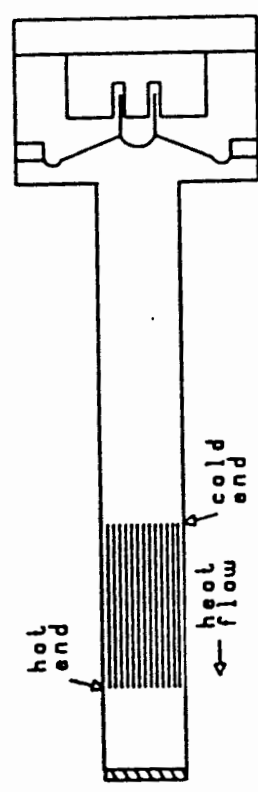

a)

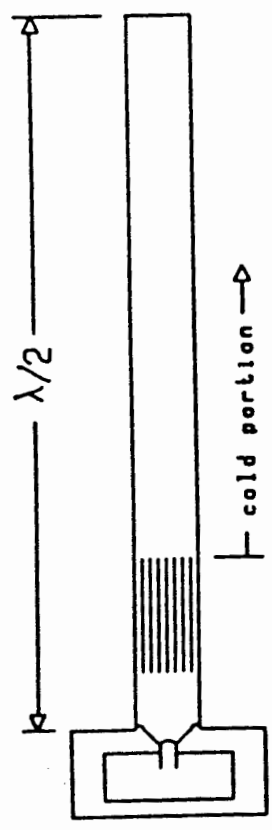

b)

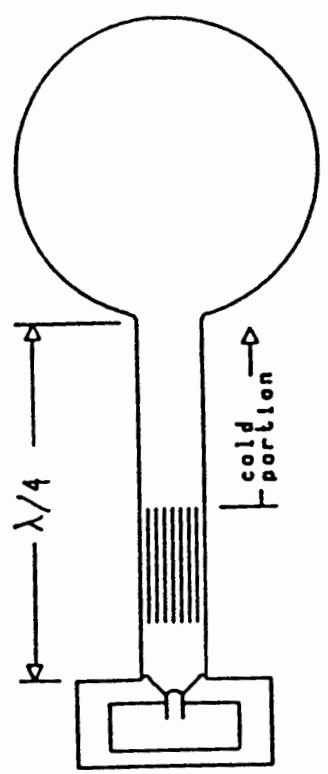

c)

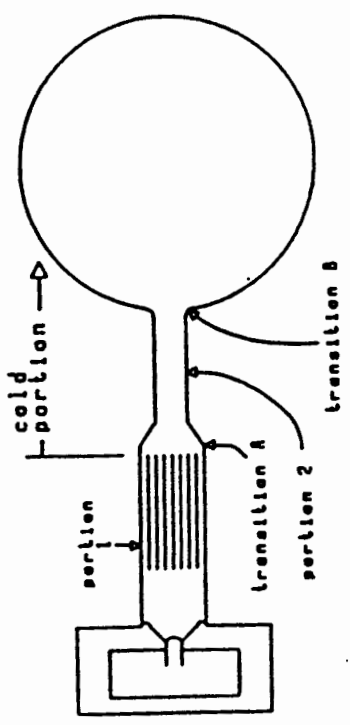

d)

Figure $6^{25}$ : Schematic of four possible resonator geometries 
Two methods to change this and improve the efficiency are possible. Since there is a standing wave set up in the tube, the placement of the driver is not critical. Thus, the system can be driven from the closed end at the pressure antinode. The cold end can now be thermally isolated and insulated. However, the geometry of the resonator has to be modified since the acoustic losses at the open end would be too large. The length of the tube can be doubled resulting in a half wavelength resonator with two closed ends (figure $6 \mathrm{~b})$ and pressure antinodes at either end or a sphere can be attached to the open end (figure 6c). This diminishes the dissipative losses considerably, and retains the property of an open end.

The researchers in Los Alamos developed an improved version of figure $6 \mathrm{c}$ where the tube underneath the stack tapers to a smaller diameter (figure 6d). The advantage can be seen both mathematically and experimentally by analyzing the thermal and viscous resonator losses. It eventually reduces to determine a so- called quality factor $\mathrm{Q}$ and then to compare it for different resonator geometries.

The factor $e^{-\mathrm{i} \omega t}$ in equation (5) and (6) must be replaced by $e^{-\frac{\beta}{2} t} e^{-\mathrm{i} \omega t}$, where $\beta$ is a damping factor describing the exponential decay of the amplitude of the oscillation. It is assumed to be small in eqn. (5) and (6). The quality factor $Q$ is defined by ${ }^{26}$

$$
Q=\pi \ln \left(-\frac{X_{n}}{X_{n+1}}\right)=\frac{\omega}{\beta} \approx \frac{\omega}{f_{2}-f_{1}}
$$

where $X_{n}$ and $X_{n+1}$ are the amplitudes of the oscillation at two subsequent maxima (separated by one full period length), $f_{1}$ and $f_{2}$ the frequency where the amplitude has dropped by the factor $\frac{1}{\sqrt{2}}$ and $\omega$ the resonance frequency. Experimentally, formula (42) can easily be verified. The theoretical treatment found in the literature goes the following way: $Q$ can be obtained by the following formula27: 


$$
Q=\frac{\omega E_{s t}}{\dot{E}}
$$

where $E_{s t}$ is the energy stored in the resonance and $\dot{E}$ is the average rate of dissipation. $E_{s t}$ can be obtained by integrating the time averaged acoustic energy density 28 over the volume of the resonator. $\dot{E}$ can be calculated by integrating thermal and viscous surface dissipation over the whole surface of the resonator. Applied to the different geometries of resonators, losses of the tapered tube are decreased by about $30 \%$ to the straight tube resonator which diminishes the losses of the half wavelength resonator by about one half. The resonator used in our experiments, ranks between the latter two. 


\section{CHAPTER 3: Experimental Setup and Construction}

\section{A. The Driver}

The simplicity in the construction consists of the usage of a regular, though slightly modified high fidelity midrange loudspeaker ${ }^{29}$. A speaker with high power capabilities had to be chosen. This is realized by speaker with a ferrofluid in the magnet gap and the voice coil wrapped around an aluminum cylinder. The main modification was the cutoff of the soft dome membrane. Instead, an aluminum cone machined in a lathe with a moving mass of 2.2 grams was glued in place. It tapers from a diameter of $5.08 \mathrm{~mm}$ where it is attached to the voice coil of the speaker down to $2.54 \mathrm{~mm}$ where the end is closed with a slight taper and a small flat in the middle. The wall thickness is $0.3 \mathrm{~mm}$. This change was due to adapting the mechanical impedance, an expression for stiffness (imaginary part) and damping (real part) of the driver, to the high acoustical impedance of the fluid at this location. In other words, the cone had to withstand the comparatively high dynamic pressure differential at the pressure antinode.

Another surround (a flexible connection between the cone and the housing) was made to close up the upper end. It consisted of a rubber like gastight fabric. It was epoxied to the cone where the slight taper starts and attached by screws and a washer to the driver's housing. It is pushed somewhat together to allow the cone to move freely. The driver is bolted to the machined aluminum housing and heat sink. A bore with the same taper as the cone was machined to the end joining the resonator tube. After placing the speaker into the housing, the surface of the housing and the cone were nearly level. The flat of the cone stuck out slightly. Care was taken to make a good thermal connection between the magnet and the housing to remove the excess heat. An aluminum lid with an O-ring 
closed the housing gastight from the top.

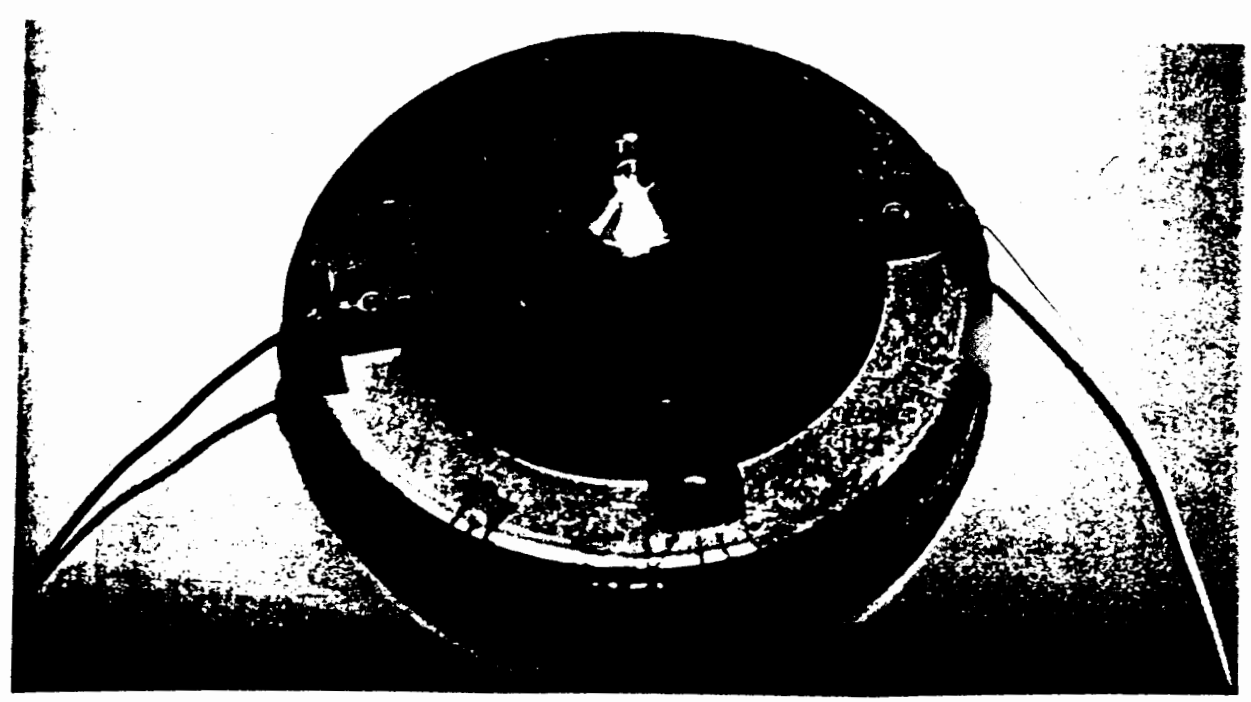

Figure 7: Picture of the modified speaker with the aluminum cone replacing the soft dome

\section{B. The Heat Exchangers}

The heat exchangers were made from copper meshes soldered on a machine made groove on the copper tube. Three screens have been used for the hot heat exchanger with a total thickness of $2.4 \mathrm{~mm}$ and two for the cold heat exchanger with a total thickness of 1.6 $\mathrm{mm}$. The screens were precisely aligned. The dimensions of the heat exchangers were chosen to be different since the hot heat exchanger has to conduct much more heat than the cold heat exchanger. Care was taken to establish a good thermal contact to the copper tube for a good heat supply and removal. 


\section{The Stack}

In contrast to the theoretical treatment, a spiral roll was used instead of a stack of parallel plates. It consists of a approximately $250 \mathrm{~cm}$ long, $80 \mathrm{~mm}$ wide and $0.12 \mathrm{~mm}$ thick flexible plastic strip. This strip is wound around a a plastic rod, that is $80 \mathrm{~mm}$ long and 8 $\mathrm{mm}$ in diameter. The spacers were made from regular $0.44 \mathrm{~mm}$ diameter fishing line. A jig was machined with grooves on either side to glue and align the spacers both vertically and parallel on the plastic strip.

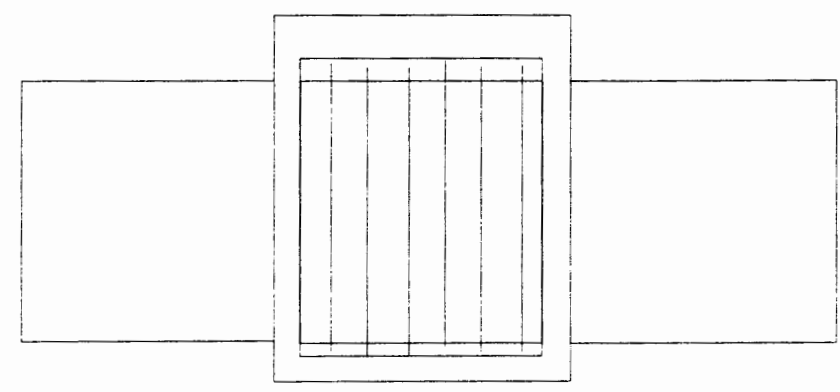

figure 8: String alignment jig and section of the plastic film

The fishing line was fastened by a bolt to one end, then laced back and forth by making use of the grooves and then bolted to the other end. 10 lines could be glued to the strip at the same time spaced $8 \mathrm{~mm}$ apart. After lacing the jig, a spray adhesive was applied to one side of the string. A flat block fitting in the middle of the jig with a weight on it was used to press the strings firmly on the plastic strip until it sticks. The strings were cut off at the edge with a scalpel. Finally, the strip was wound tightly around the rod and pushed into the tube with a piston. 


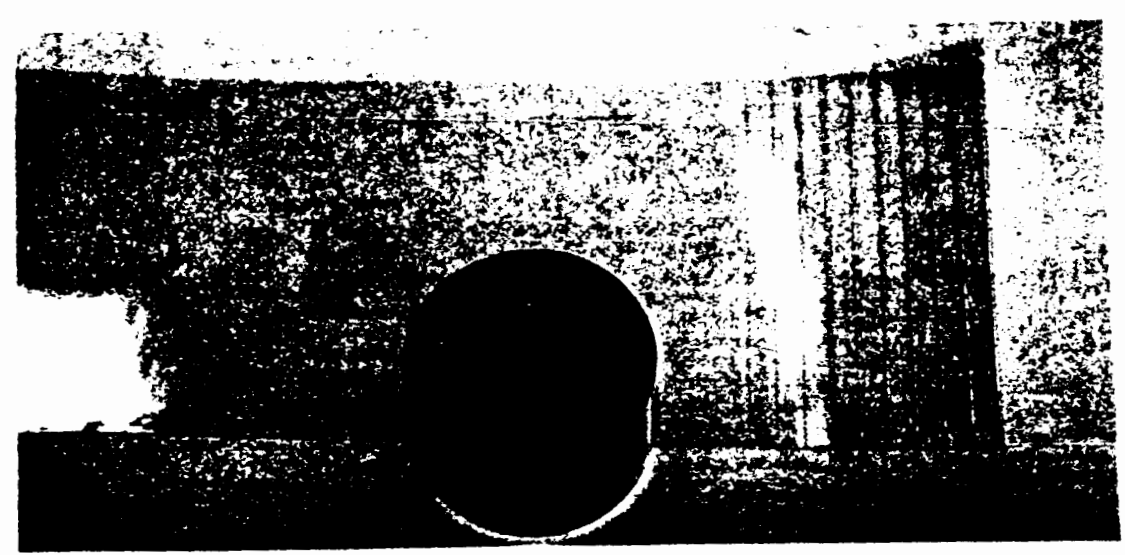

Figure 9: Picture of the rolled up stack

\section{The Resonator}

The resonator consists of a quarter wavelength tube with a cylinder shaped volume at the bottom.

For reasons of simplicity, the sphere and the neckdown (see figure 6d) were given up and replaced by a straight tube and a cylinder. The tube can be divided into three parts. The upper part containing the hot heat exchanger is made from copper to ensure good thermal conduction. It is attached to the speaker housing by a flange. Some copper tubing is wound and soldered to the flange with water flowing through to provide cooling for the 
hot heat exchanger and the speaker. This upper part is epoxied into a plastic tube. This second section contains the stack which is going to create and sustain the temperature gradient. Hence, this should be made from thermally poor conducting material. In our case this was section of Schedule 40 PVC pipe. The last section, a copper tube with the cold heat exchanger on top is epoxied from the other side into the plastic tube making contact between the stack and the heat exchanger. The other end of the tube is silver soldered onto the aluminum can which is closed with copper lids from the top and the bottom.

The cold section reaches from the cold heat exchanger to the very bottom. The large can is kept in a snug fit polystyrene box for thermal insulation from the ambient. For the rest of the cold portion, polystyrene housing was cut and placed tightly around it.

\section{E. Vacuum and Helium}

From the considerations in chapter 2.3 can be seen that helium as a working fluid is desirable. In order to change the working fluid and the pressure in the refrigerator, a vacuum system with a vacuum rotary pump and a helium pressure bottle had to be installed. The helium bottle is equipped with a regulator and a pressure gauge to adjust and measure the mean pressure in the tube. The housing and the resonator have to be evacuated and filled at the same time since there is no gas connection between them. The tubing is screwed and epoxied into the housing and resonator, respectively. 


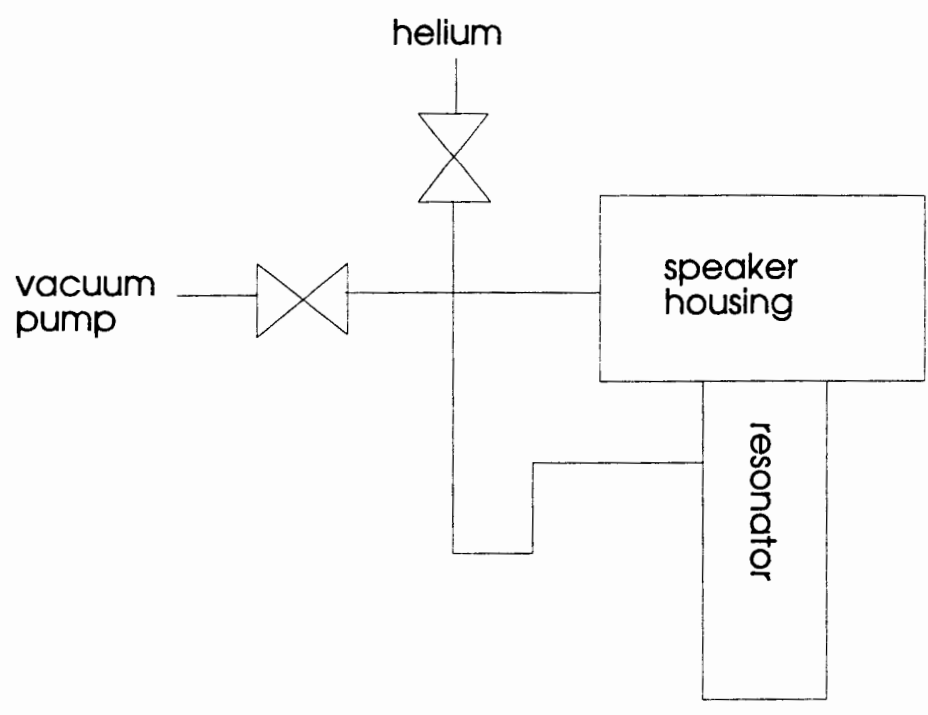

Figure 10: Drawing of the vacuum and helium filling system

\section{F. Instrumentation and Measurements}

Copper- constantan thermocouples were used for the temperature measurement. One end of each thermocouple was soldered onto the hot and the cold heat exchanger, respectively, directly on the middle of the copper screens. The wires are led through tiny holes of the tube wall. Both holes are closed with a little drop of epoxy. The reference end of the thermocouples was put in an ice- water bath in a dewar for a constant reference temperature. The obtained voltage was amplified, measured with a multimeter and converted to a temperature from the calibration curve shown in figure 11 of the thermocouple's signal versus temperature. 


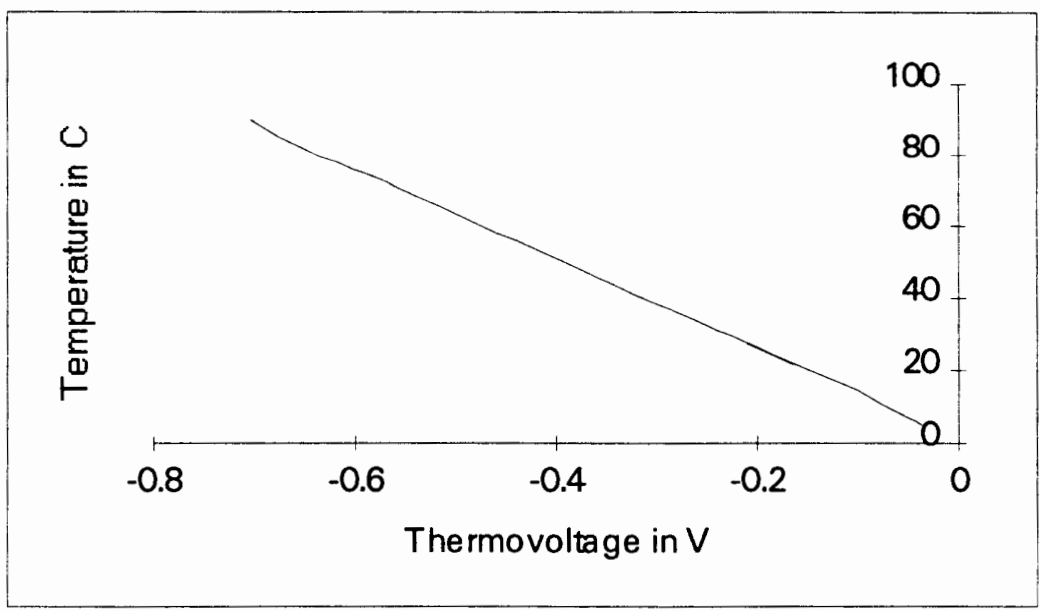

Figure 11: Calibration curve of the thermocouple in conjunction with an amplifier, with one junction in an ice bath and the other junction in water

A pressure signal was obtained by using a quartz piezo crystal glued upright onto the hot heat exchanger near the pressure antinode. The leads to the pressure transducer were introduced through a hole in the wall as well. Basically, the transducer has been used to verify the theoretical calculated resonance frequency experimentally. The resonance frequency for a pipe can be determined by the following formula considering that it is a quarter wavelength resonator.

$$
f=\frac{c}{\lambda}=\frac{1}{\lambda} \sqrt{\frac{\gamma p}{\rho_{0}}}=\frac{1}{\lambda} \sqrt{\frac{\gamma R T}{M}}
$$

with $\lambda=4 l+0.82 r . I$ is the length and $\mathrm{r}$ the radius of the resonator. The second term is a correction term ${ }^{30}$. 


\section{G. Demonstration Tube}

For demonstration purposes, a prime mover, a thermally driven oscillator has been made.

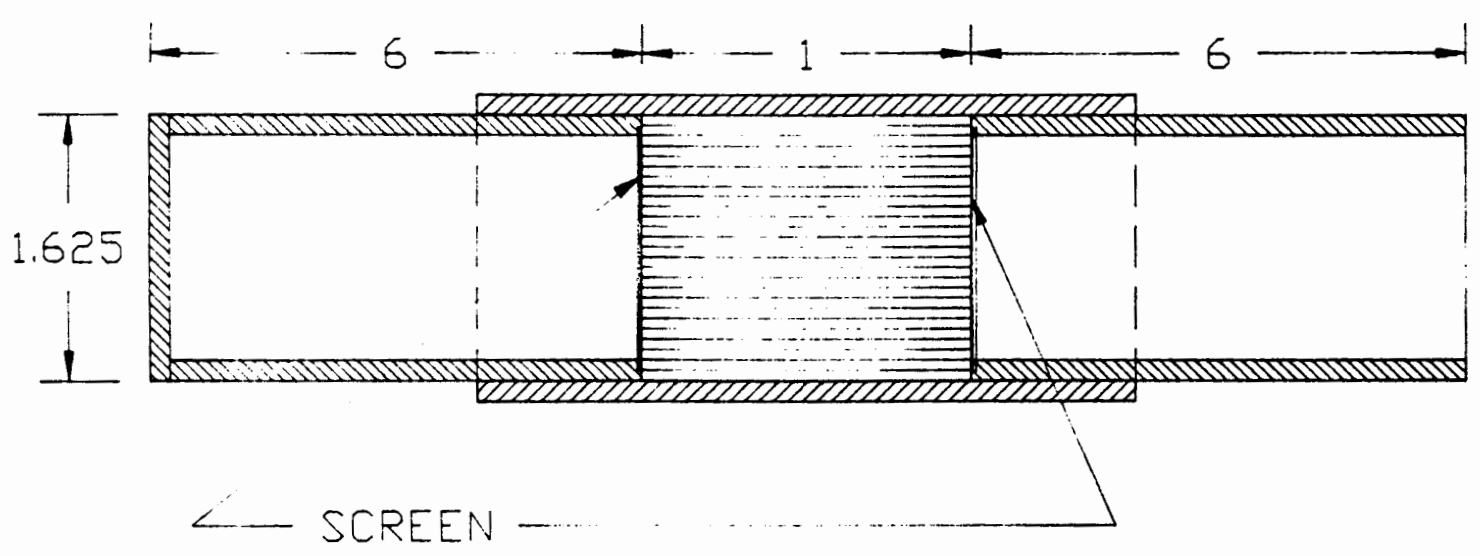

Figure 12: Drawing of a simple thermoacoustic oscillator 
As soon as a sufficient large temperature gradient has been established, it begins to oscillate spontaneously. Design and construction was kept quite simple and basically the same methods as in the refrigerator were applied.

The core parts are the stack, the heat exchangers and the resonator. Two copper tubes of equal length are used for the resonator. The same copper screens used for the heat exchangers in the refrigerator are soldered onto one end of either tube, one on each side. The stack has been made in the same way as the first one. However, the width is only $2.54 \mathrm{~cm}$. It is housed in a plastic tube. The two copper tubes were stuffed into the plastic housing until the screens make contact with the stack. One end was closed up by a copper lid. The tube has a total length of 13 in.

In operation, the open end is immersed in liquid nitrogen with the liquid level close to the heat exchanger and the closed end is kept warm by the hands. After a sufficient temperature gradient has been established it starts to vibrate and shake and after taking it out, it starts to release a sound. 


\section{CHAPTER 4: Experimental Results and Conclusions}

\subsection{Results}

The pressure signal obtained by the pressure tranducer has been measured for verifying the resonance frequency and determining the quality factor of the resonator.

The transducer turned out to work quite well. However, due to the high dynamic pressure amplitude, the signal on the oscilloscope was not sinoidial but somewhat distorted. The pressure signal of the transducer as a function of the frequency was measured for air and helium as a working fluid. This is shown in figures 13 and 14 . The resonance frequency (where the signal of the pressure transducer is greatest) can be seen in table 3. Equation (44) has been used for calculating the theoretical value.

Table 3: Calculated and experimentally determined resonance frequency for $\mathrm{T}=20^{\circ} \mathrm{C}$

\begin{tabular}{|c|c|c|}
\hline medium & $\begin{array}{c}\text { Calculated by } \\
\text { equation (44) }\end{array}$ & experimentally \\
\hline $\begin{array}{c}\text { air (fundamental } \\
\text { mode) }\end{array}$ & 267 & 281 \\
\hline $\begin{array}{c}\text { air (first mode } \\
\text { resonance) }\end{array}$ & 791 & 785 \\
\hline Helium & 762 & 805 \\
\hline
\end{tabular}




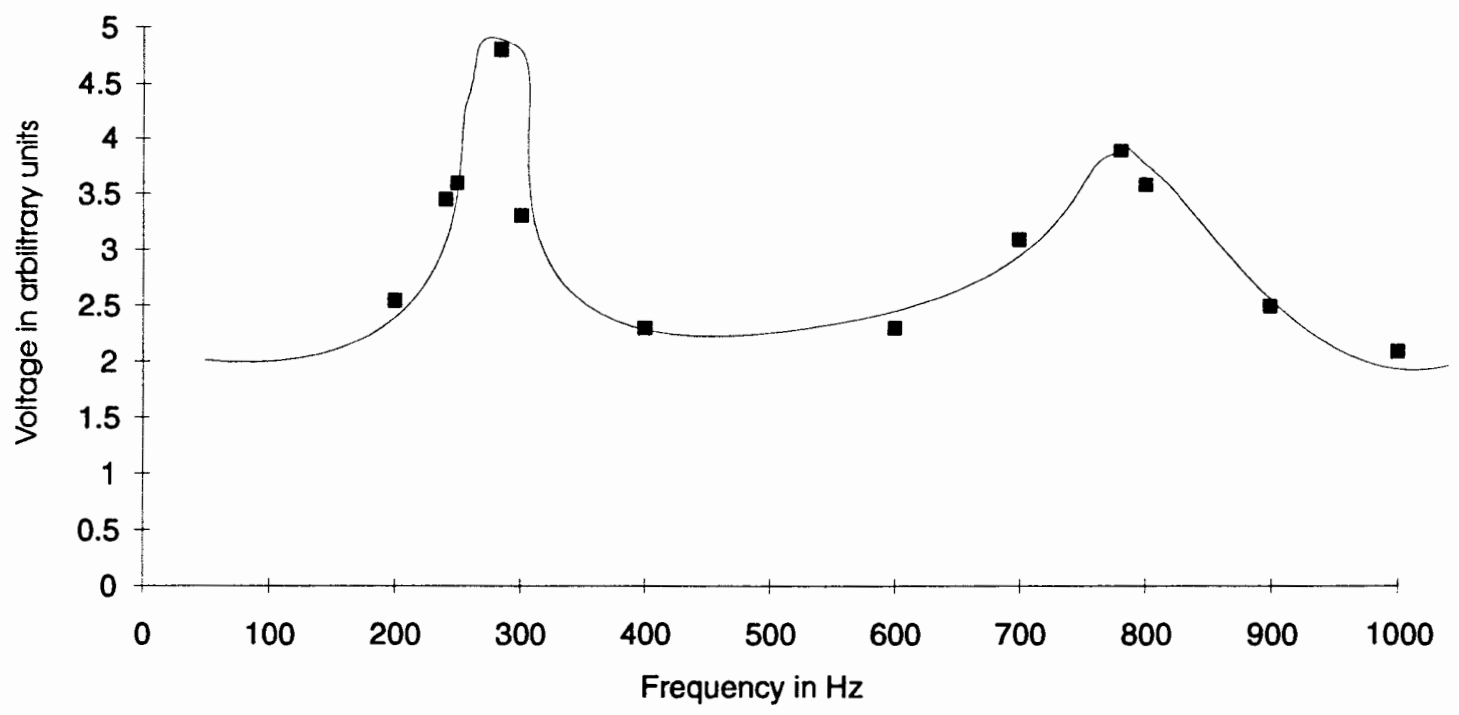

Figure 13: Voltage signal of the pressure tranducer in arbitrary units as a function of the frequency for air $\left(\mathrm{p}=1 \mathrm{~atm}, \mathrm{~T}=20^{\circ} \mathrm{C}\right)$

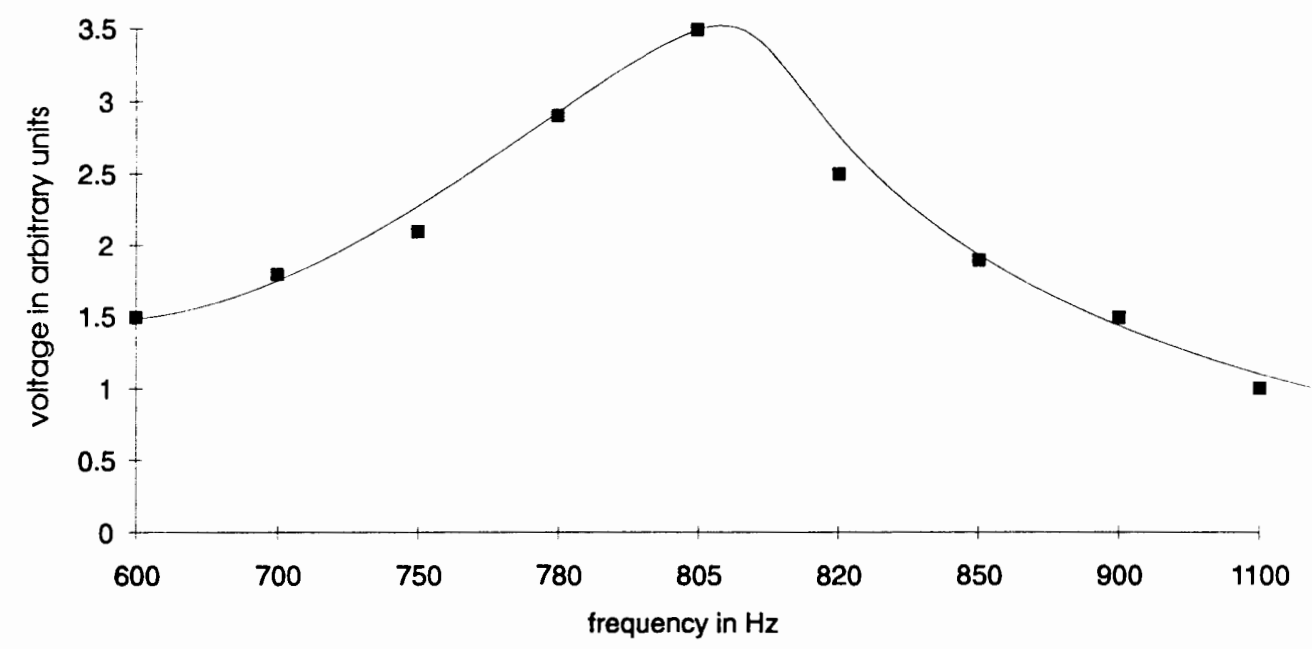

Figure 14: Voltage signal of the pressure transducer in arbitrary units versus the frequency for helium $\left(\mathrm{p}=1 \mathrm{~atm}, \mathrm{~T}=20^{\circ} \mathrm{C}\right)$

With a power output from the amplifier of $9 \mathrm{~W}$, after 10 minutes the following 
temperatures were measured with an ambient temperature of $20^{\circ} \mathrm{C}$.

The temperature of the cold heat exchanger was $16^{\circ} \mathrm{C}$ and the temperature of the hot heat exchanger was $23^{\circ} \mathrm{C}$.

\subsection{Conclusions}

The objective of this research was to build a thermoacoustic refrigerator and analyzing its basic performance.

The experimentally obtained resonance frequencies matched quite well with the calculated values. The deviations were less than $5 \%$. The two resonances in air, the fundamental mode resonance $\left(\frac{1}{4} \lambda\right)$ and the first mode resonance $\left(\frac{3}{4} \lambda\right)$ can be seen quite well.

The measured cooling effect was distinct, although quite small. According to the data reputed on the Hofler refrigerator ${ }^{3}$, a much lower temperature along with a higher cooling power would have been expected. The reasons for the comparatively poor performance are various. The main reason was the limited amount of power which could be applied to the speaker due to its repeated breakdowns. The voice coil and the surround came apart at one side. Due to the relative motions between the voice coil and the surround and due to the fact that the lead to the voice coil was in contact with the surround the lead broke off. Several attempts to fix it permanently were not successful. It broke off repeatedly, even though we ran it with minimal power ( $9 \mathrm{~W}$ in comparison to an estimated $150 \mathrm{~W}$ in the Hofler's experiment). The cold section was designed to be fairly large. It was comprised of the cold heat exchanger attached to the copper tube below as well as the large volume at the bottom. The small amount of acoustic power that could be delivered 
into the tube, was likely not enough to cool the entire section. The heat conduction in the copper tube and the brass can would in addition hinder the cooling down of the cold heat exchanger.

Finally, it can be said that we built a working acoustic refrigerator. For more elaborate results and a comparison of theory and experiment, a new speaker has to be bought or made. The instrumentation, such as the pressure transducer and accelerometer for quantitative measurement of acoustic power have to be improved and/or installed. 


\section{References}

IN. Rott, Adv. Appl. Mech. 20, 135 (1980).

2J. Wheatley, T. Hofler, ... "Understanding some simple phenomena in thermoacoustics with applications to heat engines", Am. J. Phys. 53 (2), 148 (1985).

${ }^{3}$ Th. Hofler, Dissertation, "Thermoacoustic Refrigerator Design and Performance" (1986).

${ }^{4}$ P. Storch, R. Radebaugh, J. Zimmermann, "Analytical Model for the Refrigeration Power of the Orifice Pulse Tube Refrigerator", Natl. Inst. Stand. Tech. 1343 (1990).

${ }^{5}$ Landau- Lifshitz, "Fluid Mechanics", Pergamon Oxford (1987), eqn. 64.9.

${ }^{6}$ ref. 5 , eqn. 2.3 .

${ }^{7}$ R. Sonntag, "Introduction to Thermodynamics", John Wiley \& sons New York, eqn.

12.12 .

${ }^{8}$ ref. 7, eqn. 5.15.

${ }^{9}$ ref. 7, eqn. 12.47.

${ }^{10}$ ref. 5 , eqn. 49.4 .

${ }^{11}$ ref. 7, eqn. 12.14 .

12G.W. Swift, "Thermoacoustic engines", J. of Acoustic Soc. Am. 84 (1988), 1164.

${ }^{13}$ ref. 7, eqn. 12.7 for $\mathrm{dP}=0$.

${ }^{14}$ Peter Leung, Lecture electrodynamics winter term 1994, Portland State University.

${ }^{15} \mathrm{ref} 12,1152$.

16 ref $12,1153$.

${ }^{17}$ ref $12,1156-1160$.

${ }^{18}$ ref. 5 , eqn. 15.6.

${ }^{19}$ ref $12,1176-1179$.

${ }^{20}$ ref 5 , eqn. 49.2 .

${ }^{21}$ ref. $12,1160$. 
${ }^{22}$ ref $1,138$.

${ }^{23}$ E. Verheiden, Handbook of Chemistry and Physics, 50th edition (1969), E3, E2, D125. ${ }^{24}$ ref. 23, F43.

${ }^{25}$ ref. 3 , figures 1 and 2.

${ }^{26}$ S. Bate, "Acoustical and Vibration Physics", London Eduard Arnold LTD (1966) 277-8.

${ }^{27}$ ref. $12,1162$.

${ }^{28}$ ref. 5 , eqn. 65.1 .

${ }^{29}$ Midrange high fidelity loudspeaker, Dynaudio Skanderborg Denmark, model AF 35.

${ }^{30}$ ref. $26,175$. 